\title{
Electric Field Multifractal Features in the High-Latitude Ionosphere: CSES-01 Observations
}

\author{
Giuseppe Consolini ${ }^{1, *}\left(\mathbb{D}\right.$, Virgilio Quattrociocchi ${ }^{1,2} \oplus$, Giulia $D^{\prime}$ Angelo ${ }^{1}(0)$, Tommaso Alberti ${ }^{1}{ }^{1}$, \\ Mirko Piersanti ${ }^{1} \mathbb{D}$, Maria Federica Marcucci ${ }^{1} \mathbb{D}$ and Paola De Michelis ${ }^{3}$ (I) \\ 1 INAF-Istituto di Astrofisica e Planetologia Spaziali, 00133 Rome, Italy; Virgilio.Quattrociocchi@inaf.it (V.Q.); \\ giulia.dangelo@inaf.it (G.D.); tommaso.alberti@inaf.it (T.A.); mirko.piersanti@inaf.it (M.P.); \\ mariafederica.marcucci@inaf.it (M.F.M.) \\ 2 Dip. Scienze Fisiche e Chimiche, Università degli Studi dell'Aquila, 67100 L'Aquila, Italy \\ 3 Istituto Nazionale di Geofisica e Vulcanologia, 00143 Rome, Italy; paola.demichelis@ingv.it \\ * Correspondence: giuseppe.consolini@inaf.it
}

\section{check for} updates

Citation: Consolini, G.; Quattrociocchi, V.; D'Angelo, G.; Alberti, T.; Piersanti, M.; Marcucci, M.F.; De Michelis, P. Electric Field Multifractal Features in the High-Latitude Ionosphere: CSES-01 Observations. Atmosphere 2021, 12, 646. https://doi.org/10.3390/ atmos12050646

Academic Editors: Giuseppina Nigro and Mariarosaria Falanga

Received: 29 March 2021

Accepted: 12 May 2021

Published: 19 May 2021

Publisher's Note: MDPI stays neutral with regard to jurisdictional claims in published maps and institutional affiliations.

Copyright: (c) 2021 by the authors. Licensee MDPI, Basel, Switzerland. This article is an open access article distributed under the terms and conditions of the Creative Commons Attribution (CC BY) license (https:// creativecommons.org/licenses/by/ $4.0 /)$

\begin{abstract}
In the polar ionosphere, the electric field is characterized by broadband and power law spectral densities at small/short spatio-temporal scales, which support a possible turbulent nature of the electric field fluctuations. Here, we investigate the multifractal character of the full threedimensional electric field in the polar ionosphere as recorded on board the first Chinese SeismoElectromagnetic Satellite (CSES-01). The results of our analysis prove a clear different degree of multifractality of the electric field fluctuations approaching either the polar cap trailing edge or the auroral region. The observed differences in the multifractal character are interpreted in terms of the different natures of the particle precipitation in the polar cap and in the auroral region. A possible link between the multifractal nature of electric field fluctuations, parallel to the geomagnetic field, and filamentation of field aligned currents (FACs) is established.
\end{abstract}

Keywords: auroral ionosphere; electric field; turbulence; fractals and multifractals

\section{Introduction}

Since the early studies and observations around the 1970s and 1980s, electric and magnetic field fluctuations at high-latitude ionosphere have been shown to be characterized by turbulence in the ULF (Ultra Low Frequency) and ELF (Extra Low Frequency) spectral ranges [1-3]. The turbulent character of the electric and magnetic field fluctuations manifests itself in terms of large amplitude fluctuations of physical quantities characterized by power spectral densities, following a power-law, scaling features and non-Gaussian distribution functions of small-scale increments, see, for example, [4-9], and references therein. In this framework, the results obtained by different authors in recent years are particularly interesting. For instance, electric field broadband spectra and fluctuations have been observed in the auroral regions by Kintner et al. [10]. These large amplitude and broadband fluctuations are believed to be responsible for the acceleration of auroral ions [11]. Several physical processes have been proposed to explain the broadband character of ELF electric field fluctuations, among which the possible occurrence of an intermittent turbulence [12]. The origin of this dynamics and the emergence of intermittent fluctuations have been associated by Chang et al. [13] with the occurrence of sporadic fast interactions between localized coherent plasma structures (e.g., spatial irregularities, density depletions, convective structures, electron and ion holes, etc.). In the auroral regions, such intermittent fluctuations are expected to be mainly electrostatic and transverse [10,12].

The view of intermittent turbulence proposed by Chang et al. [13] and, more generally, the scenario dealing with a near criticality dynamics has been confirmed by Tam et al. [4], who, analyzing electric field measurements from the SIERRA sounding rocket in the auroral regions, have shown that the intermittent character of the ELF electric field fluctuations in- 
creases at small scales. This result supported the view of intermittent turbulence proposed by Chang et al. [13] and, more generally, the scenario dealing with near criticality dynamics. Kozelov and Golovchanskaya [8] also supported the occurrence of strong turbulence in the auroral zone during periods characterized by northward $B_{Z}$ interplanetary magnetic field (IMF) orientations. They found that electric field fluctuations display a power-law spectral density and scaling features, thus supporting the previous scenario of intermittent turbulence proposed by Chang et al. [13] and Tam et al. [4]. Furthermore, the scaling features, quantified by the scaling index $\alpha=\zeta(2) / 2$, where $\zeta(2)$ is the scaling exponent of the 2nd structure function, are not constant and differ from those expected from classical fluid and/or magnetohydrodynamics (MHD) turbulence. Later, applying a different technique (double rank-order ROMA method) to electric field measurements in the auroral zone, Tam et al. [14] showed that contiguous multiple regimes of different physical processes exist in the range of timescales from $\tau \simeq 5 \mathrm{~ms}$ to $\tau \simeq 1 \mathrm{~s}$, and that these regimes are characterized by different scaling features, see also [15]. This more complex scenario can be interpreted in terms of a mixture of different physical processes, both MHD and kinetic, that are not characterized by the same scaling features from a physical point of view.

Similar analyses have been performed also in the polar cap regions in order to investigate the emergence of scaling features and broadband power-law spectra in the electric field capable of supporting the occurrence of turbulence, see, for example, [7], and references therein. It was found that in the polar cap regions the electric field exhibits scaling features in a range of scales from few hundred meters to hundreds kilometers and that these scaling features resemble those observed in the auroral regions. For instance, using SuperDARN measurements, Abel et al. [16,17] found that the electric field in the polar cap displays scaling features over a wide range of scales up to $1000 \mathrm{~km}$. They suggested that these scaling properties are due to the turbulent character of solar wind [16,17]. This view was successively discussed by Golovchanskaya and Kozelov [7], Kozelov and Golovchanskaya [18], underlying the similarities of the features of electric field fluctuations in the auroral and polar cap regions, and analyzing the role played by plasma convection. In particular, Golovchanskaya and Kozelov [7] underlined that in the polar cap regions the electric fields significantly correlate with the IMF $B_{Z}$ component and solar wind transfer function, which control the magnetospheric-ionospheric plasma convection, while there is no clear correlation between the electric field and IMF fluctuation fields. These results support the view of a turbulent character of the electric field also in the polar cap, whose origin could be due to convective plasma velocity shears in the Region 0 of field-aligned currents (FACs) [7]. Thus, electric fields in the polar ionosphere display a very similar turbulent nature in both auroral and polar cap regions.

The first satellite (CSES-01) of the constellation scheduled for the Chinese SeismoElectromagnetic Satellite (CSES) mission [19] was launched in February 2018. This satellite is equipped with a large set of instruments including an electric field detector (EFD) [20] with a high sampling frequency (up to $5 \mathrm{kHz}$ ) and a Langmuir probe (LP) [21]. CSES-01 flies on a Sun-synchronous orbit at $\sim 500 \mathrm{~km}$ of altitude, that is, in the topside F2 ionosphere. Although the satellite was originally designed to study possible correlations between seismic events and iono/magnetospheric perturbations, it allows to accurately investigate fine features of the electric and magnetic field fluctuations in the ionosphere for other purposes as well. CSES-01 satellite is generally operative in a limited range of geographic latitudes, typically $\mid$ Lat $\mid<65^{\circ}$, so that it is only occasionally operative in polar regions. Furthermore, due to the asymmetry of the geomagnetic dipole configuration there is a higher probability to explore the Southern polar ionosphere than the Northern one.

Here, we examine the scaling features of the full electric field vector $\mathbf{E}(t)$ measured by CSES-01 satellite during a crossing of the Southern auroral region when CSES-01 was partially operative. In detail, we analyse the spectral and multifractal features of the electric field components in the minimum variance reference plane using a direct method for evaluating the singularity spectrum [22,23]. A particular attention is also given to the vertical electric field component ( $E_{z}$ in the GEO reference one), which may indirectly 
provide information on the small scale structure of FACs. The major novelty of our study lies in the investigation of the 3D electric field fluctuations over a wide range of frequencies from DC to $2.5 \mathrm{kHz}$. This allows us to disentangle the occurrence of different dynamical regimes at different frequencies/timescales allowing to explore also the anisotropy of the fluctuations and providing new insights in the turbulent character of plasma properties and dynamics in the polar ionosphere.

The work is organized as follows: Section 2 describes the data used in the work and some simple preliminary analysis; Section 3 is devoted to the discussion of the analysis methods; Section 4 reports the results; finally, Section 5 is devoted to the discussion and conclusions.

\section{Data Description}

We consider electric field data recorded by EFD instrument [20] on board CSES-01 satellite in the frequency band from DC to $2.5 \mathrm{kHz}$ (sampling frequency $5 \mathrm{kHz}$ ) during a crossing of the Southern auroral region occurred on 11 August 2018 from 21:41 UT to 21:45 UT. The reference system of the electric field measurements is the geographical (GEO) one. Taking into account that the satellite speed is of the order of $8 \mathrm{~km} / \mathrm{s}$, the very high-sampling rate of the electric field allows us to investigate spatial scales starting from $2 \mathrm{~m}$ upwards. Indeed, if we indicate with $v_{S}$ the satellite speed then the link between the spatial $(\ell(\tau))$ and the temporal scales $(\tau)$ is $\ell(\tau) \simeq v_{s} \tau$. This relation is very similar to the usual Taylor's hypothesis used in turbulence to convert temporal scales into spatial ones, but the reasoning for this correspondence is slightly different. The correspondence between temporal and spatial scales is, in fact, possible under the assumption that most of the electric field fluctuations are essentially electrostatic and transverse $[12,24,25]$ and that the satellite speed is much larger than the movements of the turbulent fluctuations so that the temporal fluctuations are principally the effect of Doppler-shifted and stationary spatial variations. However, due to some possible instrumental noise we limit our analysis of the scaling features to scales larger than $20-40 \mathrm{~m}$.

In the selected time interval, the CSES-01 satellite is in its ascending orbit near 02:00 MLT, as shown in Figure 1 where the satellite trajectory is shown in relationship with the auroral display occurring at 21:45 UT as observed by Special Sensor Ultraviolet Spectrographic Imager (SSUSI) on board of DMSP-F17 satellite [26,27]. During this time interval CSES-01 is partially crossing a region where auroral precipitation is going on. The selected time interval is, indeed, characterized by a certain level of geomagnetic activity due to an auroral substorm occurred in consequence of a southward turning of the IMF $B_{z}$ component. During the selected period the preliminary auroral geomagnetic indices are $A E>500 \mathrm{nT}$ and $A L<-300 \mathrm{nT}$, respectively (please refer to real time AE-indices at WDC for Geomagnetism, http:/ /wdc.kugi.kyoto-u.ac.jp/aedir/, accessed on 29 March 2021).

The occurrence of auroral display is also confirmed by the $\mathrm{H}$ and $\mathrm{Z}$ components recorded at the Mawson (MAW) ground magnetometer station located in Antarctica as shown in Figure 2 (data from INTERMAGNET, http:/ / www.intermagnet.org/, accessed on 29 March 2021). The station is located within auroral latitudes at magnetic coordinates $\left(70.3^{\circ} \mathrm{S}, 90.3^{\circ} \mathrm{E}\right)$. The two magnetic components reveal the location of the station with respect to the auroral electrojet current systems during the crossing of the satellite. In the interval 20:00-24:00 UT the H component, which points toward magnetic north, shows an evident decrease. It is due to the presence in the ionosphere of an horizontal current flowing westward, which generates on the ground an horizontal magnetic field opposite to the main magnetic field direction. At UT 21:40, the station is located at around 23:00 MLT. Thus, in the analyzed period, the station is near the region crossed by satellite and is located underneath the westward auroral electrojet. However, it is not exactly located below the peak of the electrojet. Indeed, when a ground station is located directly below the peak of the electrojet, most of the ground perturbation is recorded in the $\mathrm{H}$ component with minimal or no perturbation along the $\mathrm{Z}$ (vertical) component. When, as in our case, there is a magnetic perturbation both in the $\mathrm{H}$ and $\mathrm{Z}$ component this means that the ground station 
is located, according to the increase or decrease of the $\mathrm{Z}$ component, either poleward or equatorward of the electrojet peak although it is still underneath the bulk of it. Thus, the selected ground magnetometer station permits confirmation of what is recorded by the instrument on board the DMSP and is reported in Figure 1.

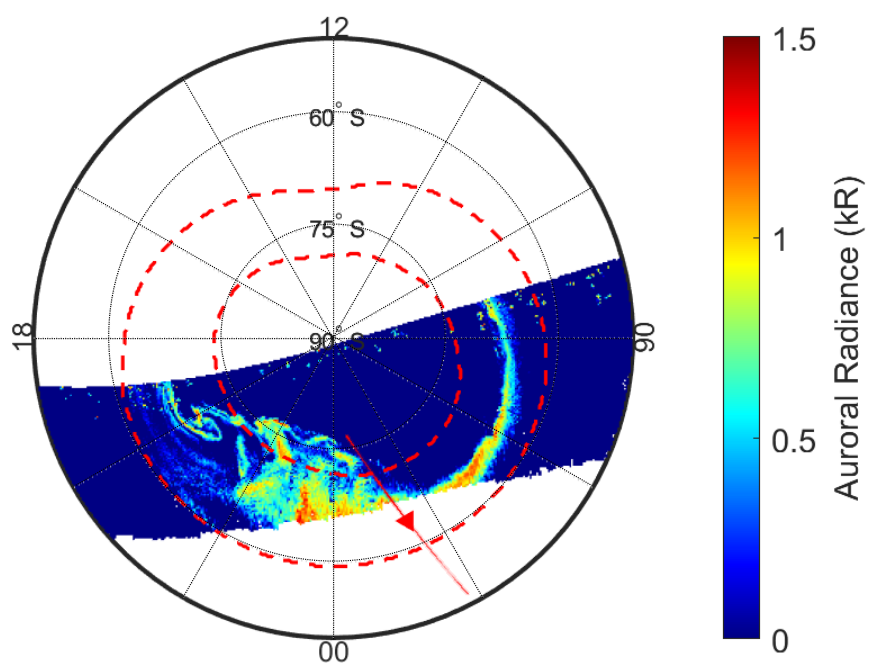

Figure 1. The CSES-01 trajectory for the selected time interval from 21:41 UT to 21:45 UT on 11 August 2018. The auroral display refers to observation by SSUSI instrument on board DMSP-F17 satellite at 21:45 UT. The red dashed curves refer to the upper and lower boundary of the auroral oval. The red curve refers to CSES-01 orbit. Reference system is AACGM (Lat, MLT) coordinate system.

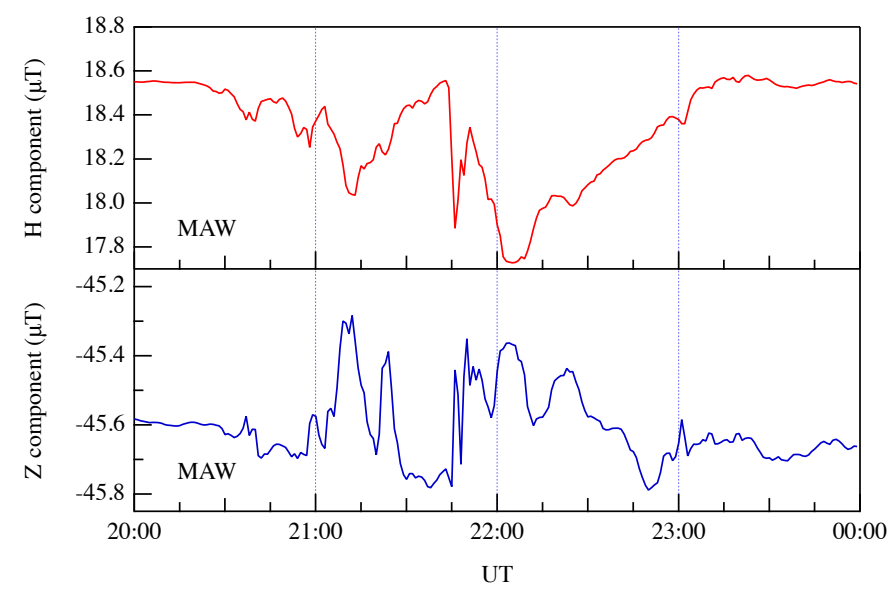

Figure 2. The ground measurements of $\mathrm{H}$ and $\mathrm{Z}$ magnetic field components at the MAW observatory.

As a first step, we remove, from each component of the $\mathbf{E}$, the motional electric field, $\mathbf{E}_{s}=\mathbf{v}_{s} \times \mathbf{B}$ where $\mathbf{v}_{s}$ is the satellite velocity, due to the spacecraft motion inside the geomagnetic field, $\mathbf{B}$ [28].

Figure 3 reports the electric field components, corrected for the $\mathbf{E}_{s}=\mathbf{v}_{s} \times \mathbf{B}$, as they results from the CSES-01 EFD measurements.

In this time interval, we selected two specific sub-intervals characterized by quasistationary conditions:

- Interval \#1: a sub-interval from 21:41:39 UT to 21:42:49 UT when the satellite is at latitude AACGM Lat $>69^{\circ} \mathrm{S}$. In this interval the electric fluctuations are small being characterized by a total variance $\sigma_{E}^{2} \sim 100 \mathrm{mV}^{2} / \mathrm{m}^{2}$;

- Interval \#2: a sub-interval from 21:43:14 UT to 21:44:39 UT when the satellite is at latitude $62^{\circ} \mathrm{S}<A A C G M$ Lat $<67.5^{\circ} \mathrm{S}$. In this case the fluctuation field is more intense being characterized by a total variance $\sigma_{E}^{2} \sim 3600 \mathrm{mV}^{2} / \mathrm{m}^{2}$. 
In choosing these two intervals we have also taken into consideration the associated spurious fluctuations due to the switch-on of the EFD instrument (the first part of the signal reported in Figure 3) and the quasi-homogeneity of the fluctuation field.

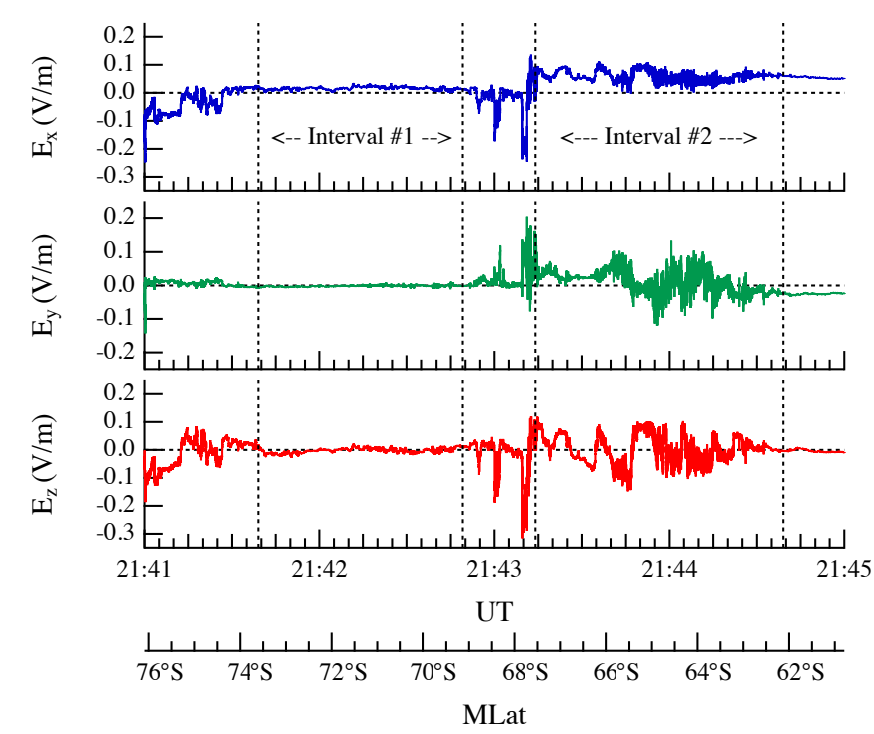

Figure 3. Electric field measurements, corrected for the $\mathbf{E}_{s}=\mathbf{v}_{s} \times \mathbf{B}$, collected by CSES-01 EFD instrument. The three components are in the geographical (GEO) reference system. The vertical dashed lines enclose the two selected time intervals considered in this study (see text for more details).

Figure 4 reports the two selected parts of CSES-01 trajectory superposed to the concurrent maps of the ionospheric convection as reconstructed from the Super Dual Auroral Radar Network (SuperDARN) observations in the Southern hemisphere (relevant references for SuperDARN are Greenwald et al. [29], Chisham et al. [30] and Nishitani et al. [31]). The ionospheric convection maps are created by the Radar Software Toolkit 4.2 [32] based on the potential mapping technique by Ruohoniemi and Baker [33]. The statistical model of Thomas and Shepherd [34] is used where radar observations are missing. The ionospheric convection for the selected intervals is characterized by two large scale convection cells with anti-sunward flows at high latitude and sunward flows at lower latitudes, in the dawn and dusk sector. The convection pattern is slightly rotated anticlockwise, as it is typical for the southward and dawnward IMF condition of the period under study [35]. Looking at the position of the trajectories with respect to the two convection cells in Figure 4 and to the auroral emission pattern in Figure 1, it appears that the satellite crosses different regions during the two time intervals. In the first interval, CSES-01 satellite is essentially near the trailing edge of the polar cap, in a region where the plasma motion could be characterized by strong shear flows. During the second time interval, CSES-01 is in the auroral region where strong particle precipitation, associated with FACs, can be present. Interestingly, during the second interval the satellite track is associated with a region of suppressed flow, already observed inside the aurora in past studies (see, e.g., Yeoman et al. [36]).

The first step of our analysis is the evaluation of the maximum fluctuation direction and the dimensionality of the fluctuation field. This is done applying the minimum variance analysis (MVA), which permits to obtain the eigenvalues, $\lambda_{i}$, and the eigenvectors, $\mathbf{e}_{i}$, associated with the covariance matrix $\hat{\Sigma}^{2}$. This approach is motivated by the fact that there is no a physical reason to prefer the geographic reference frame in which the electric field components are measured. Using the eigenvalues spectrum, it is possible to compute the dimensionality $\mathcal{D}$ of the fluctuation field according to the following expression, 


$$
\mathcal{D}=\frac{\sum_{i} \lambda_{i}}{\max \left\{\lambda_{i}\right\}}
$$

Form MVA we obtain $\mathcal{D}_{1}=1.26$ and $\mathcal{D}_{2}=1.28$ for the 1 st and 2 nd selected time interval, respectively. This result suggests that we are essentially dealing with a 1-dimension fluctuation field in both selected time intervals. Furthermore, the largest fluctuations are observed mostly along the vertical direction, being the $\mathbf{e}_{\max } \cdot \hat{\mathbf{z}} \simeq 0.9$.

Concerning the minimum variance direction, this is mainly associated with the $\hat{\mathbf{y}}$ direction in the time interval $\# 1$ and $\hat{x}$ direction in the time interval $\# 2$.
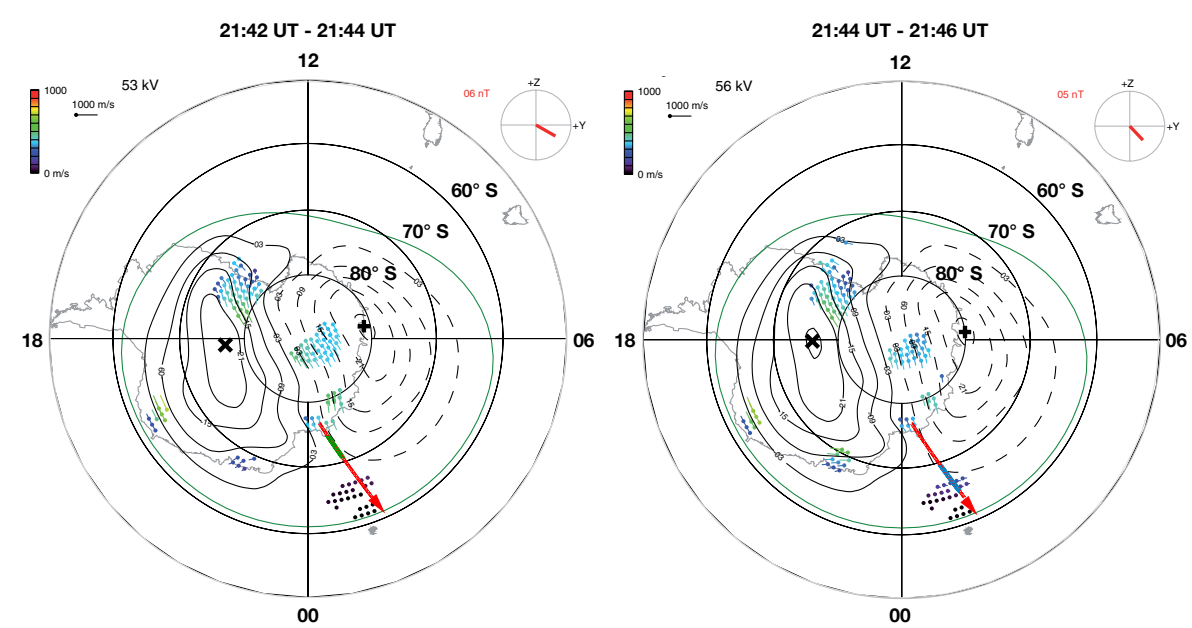

Figure 4. The CSES-01 trajectory for the two selected time intervals superimposed on the instantaneous convection cells as reconstructed by SuperDARN observations in Antarctica. The green and cyan parts of CSES-01 trajectory (red line) refer to interval \#1 and interval \#2, respectively. Reference system is AACGM MLat-MLT.

Figure 5 shows the electric field components in the minimum variance reference frame for the two time intervals. We note that the electric field fluctuations show a more homogeneous character in the first time interval, while, in the maximum variance direction, the electric field component is characterized by large fluctuations and by the occurrence of several switchbacks in the case of the second time interval.
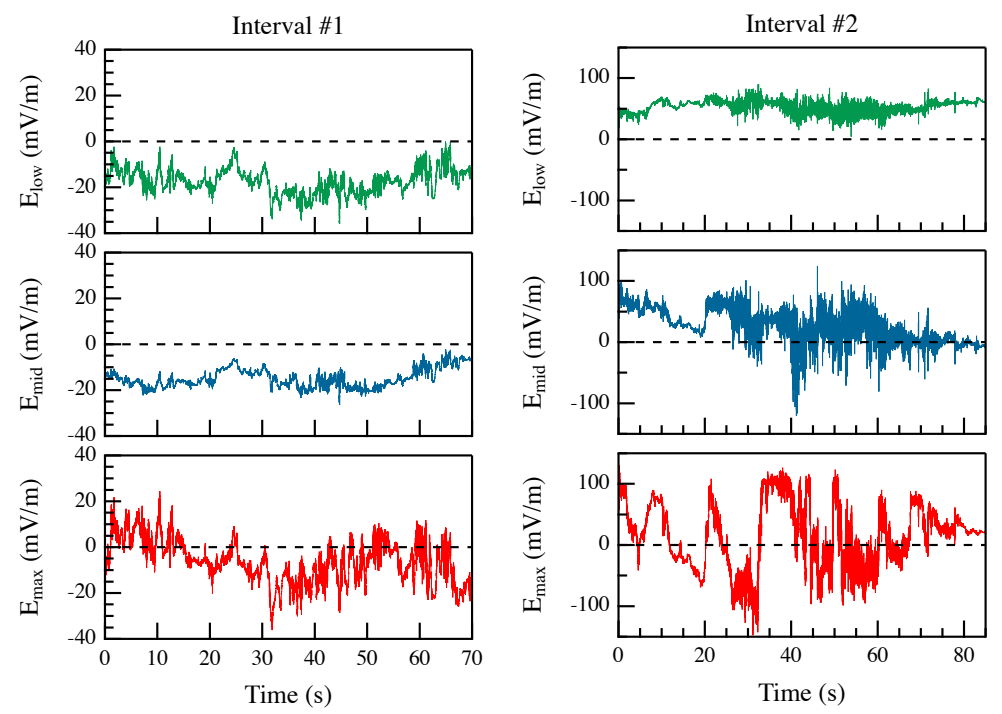

Figure 5. The electric field components in the minimum variance reference frame for the two selected time intervals. From top to bottom the components are along the minimum, the middle and the maximum variance directions, respectively. 


\section{Methods}

Several statistical methods have been proposed to investigate the scaling features and self-similarity properties of mathematical and physical objects and signals, among which the multifractal formalism is surely one of the most powerful. From a very general mathematical point of view, a multifractal object can be thought as a fractal measure defined on a fractal domain/set, that is, multifractality emerges from the interplay of two families of singularities. Paraphrasing Mandelbrot [37], another way to define a multifractal object is that of a complex fractal structure whose scaling features are no longer characterized by a single number, the fractal dimension, but by an infinite number of dimensions. The idea of the multifractal formalism is, indeed, to characterize the self-similarity of a fractal object by a hierarchy of generalized dimensions, $D_{q}$, or the so called multifractal singularity spectrum, $f(\alpha)$, which provides the fractal dimension of the set of singularities of strength $\alpha$ [38].

The evaluation of the singularity spectrum can be attained by different methods: the standard multifractal analysis [38,39], the wavelet-based multifractal analysis [40], the structure function analysis [41], and the direct determination of singularity spectrum [22,23]. These methods approaches the evaluation of the multifractal singularity spectrum in different ways which can be not adequate to our aims. For instance, the standard multifractal analysis estimates the singularity spectrum via a Legendre transformation from the partition function scaling exponents, the wavelet-based multifractal method makes use of a non physical basis (the wavelets) to estimate the spectrum, and the structure function analysis presents some difficulties in correctly estimating the spectrum at negative moments. The method proposed by Chhabra and Jensen [22] allows to determine directly the singularity spectrum from data in the real space. For this reason, we chose this method for the analysis of the electric field fluctuations.

We investigate the scaling features and, in particular, the multifractal features of the electric field fluctuations in the maximum variance direction and in the plane of minimum/medium variance directions, applying the multifractal analysis introduced by Chhabra and Jensen [22] (see also Chhabra et al. [23]). Let us, however, start by briefly resuming the singularity spectrum method $[22,23]$. The method introduced by Chhabra and Jensen [22] allows us to compute directly from data the multifractal singularity spectrum $f(\alpha)$ bypassing the Legendre transformation from the generalized dimensions $D_{q}$, as, for instance, it is done in the canonical multifractal analysis [39]. This method is particularly suitable when one deals with actual experimental data from chaotic signals whose underlying dynamics is not known. The method can be summarized as follows:

Let be $\epsilon(x)$ an experimental measure, defined over a set $\mathcal{S}$. Consider a partition of the set $\mathcal{S}$, which consists of elementary boxes of size $\ell$, and for each box $i$ compute the associated probability (fraction), $p_{i}(\ell)$, of the measure. For each box, it is possible to define a new normalized measure $\mu_{i}(q, \ell)$ of moment order $q$ as

$$
\mu_{i}(q, \ell)=\frac{\left[p_{i}(\ell)\right]^{q}}{\sum_{j}\left[p_{j}(\ell)\right]^{q}}
$$

where the moment order $q$ allows to explore regions of the measure characterized by a different degree of singularity, that is, the low singular regions for $q>1$ and the higher singular ones for $q<1$.

The Hausdorff dimension $f(q)$ of the measure $\mu_{i}(q, \ell)$ is given by

$$
f(q)=\lim _{\ell \rightarrow 0} \frac{\sum_{i} \mu_{i}(q, \ell) \ln \mu_{i}(q, \ell)}{\ln \ell} .
$$

Simultaneously, for each moment of order $q$ it is possible to estimate the associated singularity strength $\alpha(q)$, which is given by the following expression,

$$
\alpha(q)=\lim _{\ell \rightarrow 0} \frac{\sum_{i} \mu_{i}(q, \ell) \ln p_{i}(\ell)}{\ln \ell} .
$$


Equations (3) and (4) allow to get the multifractal singularity spectrum $f(\alpha)$ by simply plotting the Hausdorff dimension $f(q)$ as a function of the average singularity strength $\alpha(q)$ for each moment order $q$. A more accurate description of the method and its application to turbulence can be found in Refs. [22,23].

\section{Analysis and Results}

We start our analysis by performing the standard Fourier spectral analysis. In detail, we compute the trace (Tr) of the power spectral density (PSD) matrix $\hat{S}(f)$, which is defined as,

$$
\operatorname{Tr} \hat{S}(f)=\sum_{i} S_{i i}(f),
$$

where $S_{i i}(f)$ is the power spectral density of the $i$ th-component of the electric field. The results are shown in Figure 6 for the two selected time intervals.

In both time intervals the PSD is characterized by a power-law behavior $\operatorname{Tr} \hat{S}(f) \propto f^{-\beta}$ over a wide range of frequencies, as revealed by the broad-band character of the electric field fluctuations. At higher latitudes (i.e., the first time interval) we find a power-law scaling for $f<20 \mathrm{~Hz}$, while at lower latitudes (i.e., the second time interval) the power-law character of the spectrum extends up to $100 \mathrm{~Hz}$. In addition, the two time intervals are characterized by slightly different spectral exponents: $\beta \simeq 2$ in the first time interval and $\beta \simeq 5 / 3$ in the second time interval. These spectral features are commonly observed in the high-latitude ionosphere $[1,3,42,43]$ in terms of power-law scaling. In addition, other interesting spectral features emerge in both time intervals as the occurrence of a knee between $10 \mathrm{~Hz}$ and $100 \mathrm{~Hz}$ in the first time interval and the emergence of a peak near $f \sim 500 \mathrm{~Hz}$ in the second one. The knee, also previously observed in literature [44], can be explained in terms of the typical plasma velocity shear length, which is of the order of 200-500 m [44].

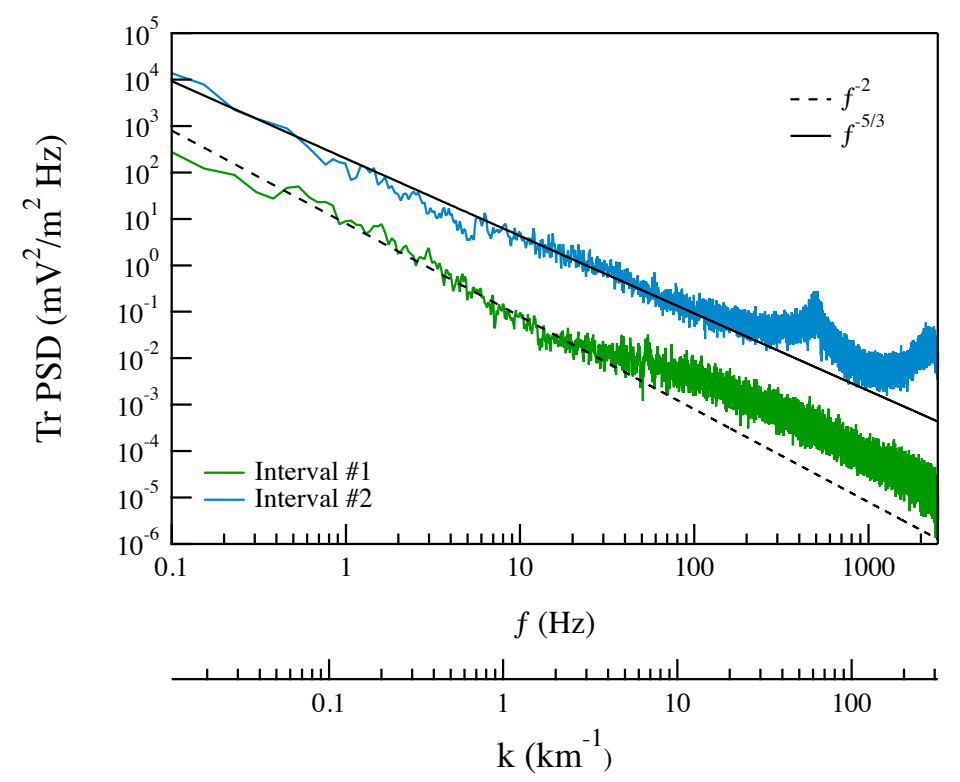

Figure 6. Trace of the power spectral density (PSD) of the electric field in the two selected time intervals. The solid and dashed lines refer to power laws $f^{-\beta}$, characterized by exponents $\beta=5 / 3$ and $\beta=2$, respectively. The secondary bottom axis is computed considering the satellite speed $v_{s}$ as $k=f / v_{s}$, being $v_{s} \simeq 8 \mathrm{~km} / \mathrm{s}$.

To better characterize the spectral features, Figure 7 reports the spectral properties of the two time intervals in the maximum (Max) variance direction and in the minimum/medium (Min/Med) variance plane, respectively. We remind that the maximum variance direction is quasi-parallel to the vertical $(Z)$ direction, while the Min/Med vari- 
ance plane essentially coincides with the horizontal plane. The electric field fluctuations show some small spectral differences in the two different directions essentially in second time interval. This suggests that the physical mechanisms related to the two directions are different. Conversely, no significant differences are observed in the first time interval apart from the power spectral content.
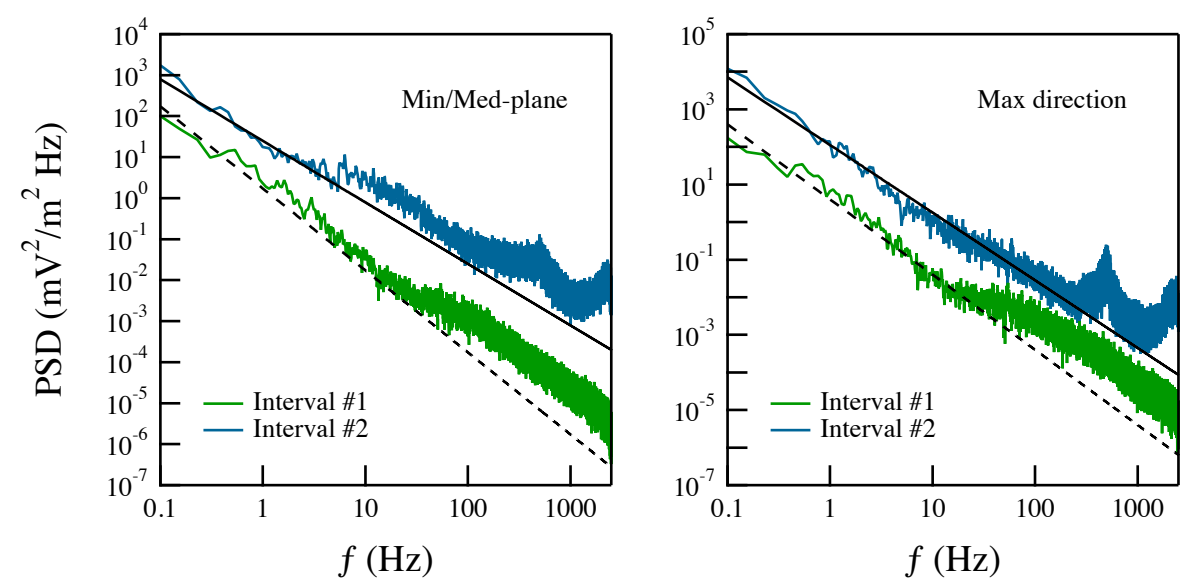

Figure 7. The PSD of electric field fluctuations in the two selected intervals in the Min/Med variance plane (eft panel) and in the maximum variance direction (right panel), respectively. The dashed and solid lines are power laws $f^{-\alpha}$ with $\alpha=2$ for dashed lines, and $\alpha=1.5$ and $\alpha=1.8$ for solid lines in left and right panels, respectively.

In order to perform the multifractal analysis according to Chhabra and Jensen [22] and Chhabra et al. [23], it is necessary to define a stationary measure $\epsilon(x)$. Being the spectral features of the analyzed time series characterized by a quasi power-law behavior with an exponent $\alpha \in(1,3)$, the time series of the two selected time intervals are nonstationary with stationary increments [45]. Thus, a stationary measure can be defined on the basis of the short time scale increments, $\delta E_{i}=E_{i}(t+\delta t)-E_{i}(t)$ where $\delta t$ is the time resolution, that is,

$$
\epsilon_{\text {Max }}(t)=\delta E_{\text {Max }}^{2}(t)
$$

and

$$
\epsilon_{\text {Min } / \text { Med }}(t)=\delta E_{\text {Min }}^{2}(t)+\delta E_{\text {Med }}^{2}(t) .
$$

The above definition of a stationary measure is commonly used in space plasma turbulence see, for example, [46]. Once we have defined a measure, we compute the measure fraction $p_{i}(\tau)$ contained in each subset $\Omega_{i}(\tau)$ of a regular partition of order $\tau$ of the interval $\Omega(T)$ of length $T$ on which the measure is computed. Thus, we get

$$
p_{i}(\tau)=\frac{\int_{\Omega_{i}(\tau)} \epsilon_{k}(t) d t}{\int_{\Omega(T)} \epsilon_{k}(t) d t},
$$

where $\epsilon_{k}$ is one of the measures defined in Equations (6) and (7).

Using the definition of the measure fraction $p_{i}(\tau)$ of Equation (8) by means of the expression in Equations (2)-(4), we can compute $f(q)$ and $\alpha(q)$ as a function of $\tau$. In detail, we compute the two quantities $\sum_{i} \mu_{i}(q, \tau) \ln p_{i}(\tau)$ and $\sum_{i} \mu_{i}(q, \tau) \ln \mu_{i}(q, \tau)$ as a function of $\tau$ and look for the existence of scaling domain plotting the two quantities versus $\ln \tau$.

Figure 8 shows the behavior of the previous two quantities versus $\ln \tau$ at different $q$ values in the case of the first time interval and for the maximum variance direction component. A good linear scaling is found in the case of two different ranges of scales. The separation scale is $\tau^{*}=75 \mathrm{~ms}$, roughly corresponding to a frequency $f \simeq 7 \mathrm{~Hz}$ $\left(k \simeq 1 \mathrm{~km}^{-1}\right)$. 


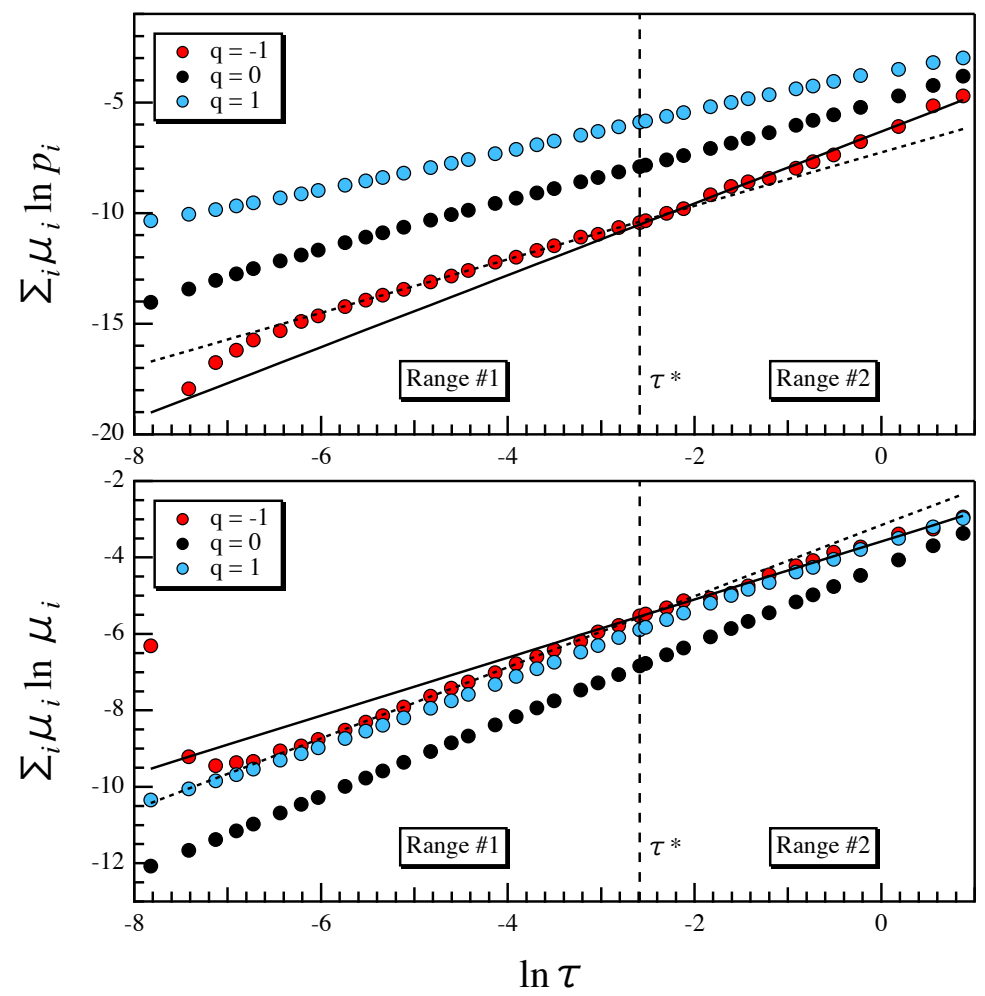

Figure 8. $\sum_{i} \mu_{i}(q, \tau) \ln p_{i}(\tau)$ (upper panel) and $\sum_{i} \mu_{i}(q, \tau) \ln \mu_{i}(q, \tau)$ (lower panel) as a function of $\ln \tau$ at different $q$ values in the case of the first selected time interval and for the the maximum variance direction component. Solid and dashed lines are linear fits. The vertical dashed line refers to the separation scale $\tau^{*}=0.075 \mathrm{~s}$ between the two different dynamical ranges.

The existence of different scaling ranges in the case of electric field fluctuations has already been found by Tam et al. [14], Tam and Chang [15]. Furthermore, the separation scale agrees with the crossover time scale $\tau=80 \mathrm{~ms}$ between Regime 1 and Regime 2 observed by Tam and Chang [15] while we are not capable of identifying the four different regimes observed in Tam and Chang [15].

Using the previous results we compute the multifractal singularity spectra in the two scale ranges for both intervals with $q \in[-4,4]$ being the total number of available measurements of $\sim 10^{5}$ points for both the two selected intervals. We start by investigating the maximum variance direction field. Figure 9 shows the singularity spectra, $f(\alpha)$, obtained by analyzing the electric field component in maximum variance direction for the two selected time intervals and for the two dynamical ranges. The results show significant differences between both time intervals and the dynamical ranges. The most significant difference is the wider character of the singularity spectrum in the range \#2 between the two intervals, which suggests that the features of the fluctuations in first time interval are more intermittent than in the second one.

This can be quantified by computing the singularity spectrum amplitude $\Delta \alpha=\alpha_{\max }-$ $\alpha_{\min }$, where $\alpha_{\min }$ and $\alpha_{\max }$ are the intersections of the singularity spectrum with the Xaxis, that is, the values for $f(\alpha)=0$. The two values of $\alpha_{\min }$ and $\alpha_{\max }$ are computed by interpolating the singularity spectrum $f(\alpha)$ using a high degree ( 5 or 7$)$ polynomial. Table 1 reports the obtained values of the singularity spectrum amplitude $\Delta \alpha$ for the two selected intervals in the two different ranges of time scales.

The electric field fluctuations along the maximum variance direction show, in both ranges, a more pronounced multifractal character in the first time interval than in the second one. This suggests that the intermittency phenomenon is more relevant in the first time interval. 

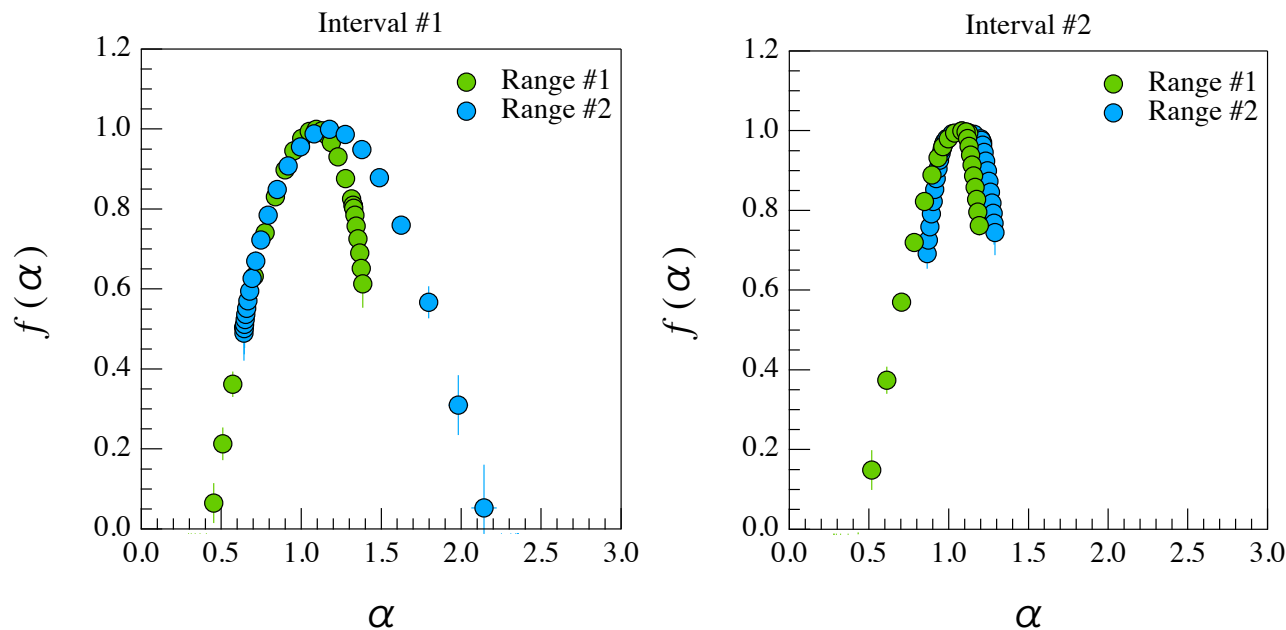

Figure 9. The multifractal singularity spectra, $f(\alpha)$, relative to the two range of scales for the two selected time intervals. The results refer to the maximum variance direction.

Table 1. The singularity spectrum amplitude $\Delta \alpha_{i}$ for the two dynamical ranges of the two intervals $\# i$ in the case of the maximum variance direction fluctuations.

\begin{tabular}{ccc}
\hline & $\Delta \alpha_{\mathbf{1}}$ & $\Delta \boldsymbol{\alpha}_{\mathbf{2}}$ \\
\hline Range \#1 & 1.145 & 1.740 \\
Range \#2 & 0.585 & 0.805 \\
\hline
\end{tabular}

The same analysis is performed in the case of the Min/Med variance plane using the measure defined in Equation (7).

Figure 10 reports the singularity spectra, $f(\alpha)$, for the two intervals in the Min/Med variance plane. As in the previous case, the spectra relative to the first time interval are wider than those for the second one. Thus, the electric fluctuation field, although characterized by a small variance, is more intermittent in the first time interval (see also data on $\Delta \alpha$ reported in Table 2). Furthermore, the degree of intermittency, as estimated by $\Delta \alpha$, is generally higher in the case of the maximum variance direction (see Tables 1 and 2).
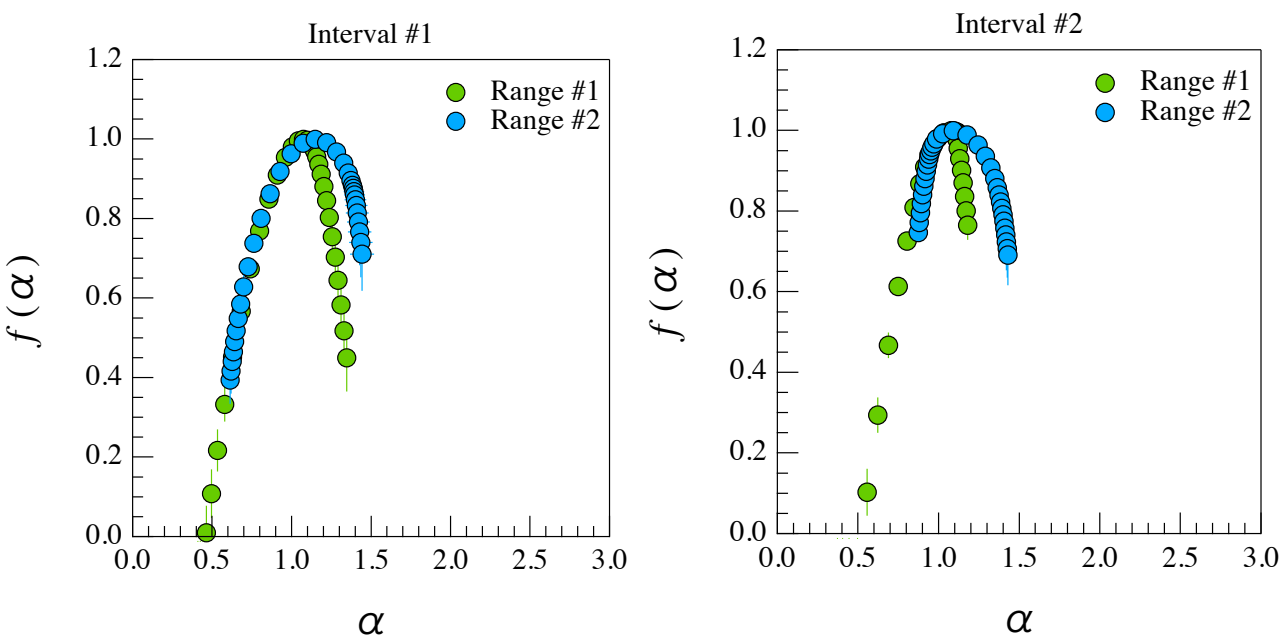

Figure 10. The multifractal singularity spectra, $f(\alpha)$, relative to the two range of scales for the two selected time intervals. The results refer to the Min/Med variance plane. 
Table 2. The singularity spectrum amplitude $\Delta \alpha_{i}$ for the two dynamical ranges of the two intervals $\# i$ in the case of the Min/Med variance plane fluctuations.

\begin{tabular}{lll}
\hline & $\Delta \alpha_{1}$ & $\Delta \alpha_{2}$ \\
\hline Range \#1 & 0.95 & 1.18 \\
Range \#2 & 0.70 & 0.78 \\
\hline
\end{tabular}

Another quantity capable of quantifying differences in multifractal singularity spectra is the skewness $S$ of the singularity spectrum which is defined

$$
s=\frac{\alpha_{\text {max }}-\alpha_{\text {peak }}}{\alpha_{\text {peak }}-\alpha_{\text {min }}}
$$

where $\alpha_{\text {peak }}$ is the position of the maximum of $f(\alpha)$. Indeed, when $s<1$ the singularity spectrum is dominated by weaker singularities and the signal is more smooth, while when $s>1$ the signal is more rough. Table 3 reports the values of the skewness $s$ for the two intervals in the two different ranges.

Table 3. The skewness of the singularity spectra amplitude $s_{i}$ for the two dynamical ranges of the two intervals $\# i$ in the case of the Max and Min/Med variance plane fluctuations.

\begin{tabular}{ccccc}
\hline & $s_{1}^{\text {Max }}$ & $s_{2}^{\text {Max }}$ & $s_{1}^{\text {Min/Med }}$ & $s_{2}^{\text {Min/Med }}$ \\
\hline Range \#1 & 0.66 & 0.49 & 0.60 & 0.52 \\
Range \#2 & 1.48 & 1.61 & 0.50 & 1.69 \\
\hline
\end{tabular}

Looking at the skewness values we notice that the first dynamical range, in both the two selected time intervals and directions, is characterized by similar values $(s<1)$ suggesting that the singularity spectrum is generally characterized by weaker singularities, so that the electric field fluctuations at these scales are expected to be more smooth. Conversely, in the second range of scales $s$ is generally larger than 1, suggesting a higher degree of roughness of the electric field fluctuations. There is however a peculiar situation in this second range of scales. The fluctuations in the Min/Med variance plane for the first interval are characterized by weaker singularities $(s<1)$. This difference could be due to the different nature of the plasma motion and particle precipitation in the two selected time intervals.

Summarizing the results of the multifractal analysis, we can conclude that there is a significant difference in the singularity spectra of the two selected time intervals and that the electric field fluctuations of the first interval display a more multifractal nature. Furthermore, the singular nature of electric field fluctuations is more marked in the maximum variance direction, a property which is common to both intervals in Range \#2. These results suggest that different physical mechanisms are working in the different polar regions generating fluctuations characterized by a different multifractal character.

\section{Discussion}

We begin by discussing briefly the observed spectral features. In addition to the slightly different spectral exponent in the low-frequency domain, the other relevant result of the spectral analysis is the emergence of a spectral break near $10 \mathrm{~Hz}$ (in both the two intervals), which is followed by a flattening of the spectrum at higher frequencies in the auroral region (Region \#2). Although this behavior can be related to the typical plasma velocity shear length [44], it also recalls what was observed in MHD turbulence simulations [47], where it was found that, in the direction perpendicular to the magnetic field, the large scale fluctuations display a steeper spectrum than the small scale ones. This was interpreted in terms of an enslaving of small-scale fluctuations to large-scale ones due to the dynamic alignment between velocity and magnetic field fluctuations [47]. Although this scenario was proposed in the case of MHD turbulence, we could conjecture that something 
similar could also occur in our case. Furthermore, as shown by Friedrich et al. [48] the dynamic alignment scenario could cause the formation of pressure depletion regions where the alignment is relevant. A similar phenomenon could occur in the auroral region, where FACs play an important role. This point clearly requires a deeper investigation that we demand to future works. However, another possible mechanism explaining the occurrence of the flattening of the spectra at frequencies higher than $10 \mathrm{~Hz}$ could be the occurrence of a less efficient energy transfer in the turbulence mechanism implying a sort of bottleneck effect at higher frequencies that implies a piling up of the fluctuation energy in the spectral interval from $\sim 10 \mathrm{~Hz}$ to $\sim 100 \mathrm{~Hz}$.

The MVA and the spectral properties of the electric field components evidenced the anisotropic character of the fluctuation field in both selected regions. The largest fluctuation variance is mainly along the vertical component suggesting that this could be due to particle precipitation and perhaps FACs.

To show that the last statement is reasonable, let us start from the generalized Ohm's law [49], that is,

$$
\vec{j}=\hat{\sigma}\left(\vec{E}+\vec{v} \times \vec{B}+\frac{1}{q n} \nabla p_{e}\right)-\left(\frac{\omega_{c e}}{v}\right) \vec{j} \times \frac{\vec{B}}{B},
$$

where $\hat{\sigma}$ is the conductivity tensor, $\vec{v}$ is the plasma fluid velocity, $\vec{B}$ the magnetic field, $\vec{j}$ the electric current density, $n q$ is the electric charge density, $\nabla p_{e}$ is the electron scalar pressure gradient, $\omega_{c e}$ the electron cyclotron frequency and $v$ the plasma collisional frequency. Here, we neglected the electron inertia term. The last term in Equation (10) is the Hall term, which can be neglected if we assume that the current density is essentially field-aligned, that is,

$$
\vec{j} \times \frac{\vec{B}}{B} \simeq 0, \Leftrightarrow \vec{j} \| \vec{B} .
$$

Furthermore, the electron pressure gradient density $\nabla p_{e}$ can be written as $\nabla p_{e}=\nabla_{\|} p_{e}+$ $\nabla_{\perp} p_{e}$, where, considering the region crossed by CSES-01, we can assume $\nabla_{\|} p_{e} \ll \nabla_{\perp} p_{e}$. Thus, the generalized Ohm's law becomes,

$$
\vec{j}=\hat{\sigma}\left(\vec{E}+\vec{v} \times \vec{B}+\frac{1}{q n} \nabla_{\perp} p_{e}\right)
$$

Specializing the terms of the last equation in the case of FAC region, that is, assuming $\vec{E}=\left\{E_{\perp}^{1}, E_{\perp}^{2}, E_{\|}\right\}, \vec{B}=\left\{\delta B_{\perp}^{1}, \delta B_{\perp}^{2}, B_{0}\right\}$, and $\vec{v}=\left\{v_{\perp}^{1}, v_{\perp}^{2}, v_{\|}\right\}$is the plasma fluid velocity, we get for the electric field parallel component the following expression,

$$
\sigma_{\|}\left[E_{\|}+\left(v_{\perp}^{1} \delta B_{\perp}^{2}-v_{\perp}^{2} \delta B_{\perp}^{1}\right)\right]=j_{\|} .
$$

Here, the conductivity tensor $\hat{\sigma}$ is assumed to be of the form,

$$
\hat{\sigma}=\left(\begin{array}{ccc}
\sigma_{\perp}^{(11)} & \sigma_{\perp}^{(12)} & 0 \\
\sigma_{\perp}^{(21)} & \sigma_{\perp}^{(22)} & 0 \\
0 & 0 & \sigma_{||},
\end{array}\right),
$$

where the components $\sigma_{\perp}^{(i, j)}$ are associated with the Pedersen and Hall conductivity and $\sigma_{\|}$ is the parallel conductivity.

Considering that the perpendicular plasma velocity is small (of the order of some kilometers per second) and the magnetic field transverse fluctuations are small $\left|\delta B_{\perp}\right| \sim 100-1000 \mathrm{nT}$ and negligible with respect to the parallel direction where the local large-scale geomagnetic field resides, that is, $\delta B_{\perp}^{1}, \delta B_{\perp}^{2} \ll B$, we get a contribution to the parallel electric field of the order of $0.1-1 \mathrm{mV} / \mathrm{m}$ by the Lorentz term in Equation (13). We can consider this contribution to the parallel electric field component negligible (we found $E_{\max } \sim 10-100 \mathrm{mV} / \mathrm{m}$ ), 
and so the previous expression in Equation (13) can be approximated at the leading order as it follows,

$$
\sigma_{\|} E_{\|} \simeq j_{\|}
$$

Thus, assuming that the magnetic field is quasi-vertical in the investigated polar regions, we may conjecture that the electric field fluctuations in the maximum variance direction are mainly related to spatial intensity variation of FACs, which flow parallel to the magnetic field. In such a scenario, we can assume that the multifractal character of the vertical component of the electric field is linked to a similar structure of FACs. Although this could appear as a simple speculation, a preliminary analysis of the FACs structure (here not shown), using Swarm satellite measurements, seems to support this view (see also Consolini et al. [50] for scaling features of FACs). The idea is that the region of FACs consists of domains of positive and negative currents structures and that this structure is hierarchical. Thus, the larger multifractal character of fluctuations along the maximum variance direction in the first time interval, being also more pronounced in the range \#2 corresponding to scales larger than $1 \mathrm{~km}$, can be related to the large-scale structure of FACs. This means that the underlying fractal support associated with FACs and particle precipitation is that corresponding to a more inhomogeneous and filamentary structure, typically due to localized current sheets flowing along the main magnetic field structure.

At smaller spatial scales, that is, below $1 \mathrm{~km}$, the multifractal/intermittent character is reduced in both time intervals, being $\Delta \alpha_{2} / \Delta \alpha_{1} \sim 1.52$ in the first time interval and $\Delta \alpha_{2} / \Delta \alpha_{1} \sim 1.38$ in the second one. This reflects a more homogeneous and regular fractal support that is typically observed when dissipation/kinetic mechanisms act and/or when the observed regime is due to processes occurring on shorter scales than those reliably observable due to instrumental noise and performances.

A similar behavior is also found in the case of the multifractal spectra of the electric field component in the minimum/medium variance direction, although the multifractal character is less marked in comparison with the maximum variance direction. Furthermore, in this case also the difference in the singular character of fluctuations between the two ranges is less relevant, being $\Delta \alpha_{2} / \Delta \alpha_{1} \sim 1.24$ and $\Delta \alpha_{2} / \Delta \alpha_{1} \sim 1.11$ in the first and second time interval, respectively. This seems to point towards a more regular underlying fractal support associated with the auroral convection cells that tends to regularize plasma motion with a sort of "confinement mechanism".

According to Tam and Chang [15] a possible explanation of the less singular character of the fluctuations at time scales shorter than $75 \mathrm{~ms}$ (spatial scales smaller than $1 \mathrm{~km}$ ) could be the occurrence of kinetic effects at small scales. Indeed, in this range of temporal and spatial scales, applying a more sophisticated analysis, the ROMA double rank-ordering technique, Tam and Chang [15] observed a more persistent character of electric field fluctuations and a rapid growing of this feature with the fluctuation amplitude that could be due to the effects of rapidly growing linear or nonlinear instabilities at small amplitudes. We can also note that the less singular character of electric field fluctuations in this range of spatio-temporal scales, as measured by the $\Delta \alpha$, is well in agreement with the smallest spreading of ROMA $s_{1}$ scaling exponents $\left(\Delta s_{1} \simeq 0.2\right)$ found by Tam and Chang [15] (please refer to Figure 9 in their paper). As stated in some previous works $[3,44,51]$ at these temporal and spatial scales a particular role could be also played by low-energy ion conics and particle injection. Furthermore, this domain could be also dominated by ion-cyclotron waves [3] and/or ion-acoustic waves. A possible origin of the observed spectral features at these scales could be due to the emergence of broadband electrostatic waves which result from the combined effects of sheared flows and field-aligned currents [3]. In such a scenario the peak observed in the maximum variance direction spectrum in the second time interval could be associated with a typical spatial scale of the filamentary structure of the FACs, which would be of the order of $20 \mathrm{~m}$. The idea is that, in the region of FACs, these filamentary currents could interact generating a sort of resonance phenomenon at the observed spatial scale/frequency. 
Conversely, at larger temporal and spatial scales, that is, $\tau>75 \mathrm{~ms}$ and $k<1 \mathrm{~km}^{-1}$, the increase of the multifractal and singular character of electric field fluctuations could be due to a decrease of the relevance of the kinetic effects. At these ranges of scales the MHD turbulence could be more relevant so that the observed fluctuation field could resemble that generated by reduced-MHD turbulence [52,53]. We note how in this domain the ROMA analysis by Tam and Chang [15] found an anti-persistent character of fluctuations and larger variability of ROMA $s$ scaling exponents $\left(\Delta s_{i} \in[0.2,0.4]\right.$ in Regimes 2,3 and 4$)$ as reported in their Figure 9.

Last but not least, in agreement with the work by Spicher et al. [54], and different from the analyses by Golovchanskaya and Kozelov [7], our results support the existence of slight differences between the multifractal features of the electric field measurements at the trailing edge of the polar cap and that in the auroral oval where the plasma dynamics is strongly affected by FACs. This different character of the fluctuations in the two regions is also supported by the different value of the skewness of the singularity spectrum relative to the Min/Med variance plane that reveals as the electric field fluctuations are characterized by weaker singularities in Region \#1 (polar cap trailing edge) than in Region \#2 (auroral region).

\section{Conclusions}

In this work, we have investigated the scaling features of the fluctuations of the full3D electric field as measured by the CSES-01 satellite in a crossing of the Southern polar ionosphere. The use of the CSES-01 measurements has allowed to investigate the electric field fluctuations over a wide range of frequencies from DC to $2.5 \mathrm{kHz}$ and to study the emergence of anisotropy both in spectral and scaling features. The analysis mainly based on the direct determination of the multifractal singularity spectrum.

The results of our analysis evidenced how the multifractal nature of the electric field fluctuations is scale dependent, that is, there is a change of the multifractal properties at a typical scale of $\tau=75 \mathrm{~ms}(\sim 1 \mathrm{~km})$. In particular, the fluctuations of the electric field display the occurrence of a weaker singularity nature at short timescales (i.e., at $\tau<75 \mathrm{~ms}$ ) and a more rough nature at long timescales (i.e., at $\tau>75 \mathrm{~ms}$ ). A similar transition was observed by Tam et al. [14] and Tam and Chang [15]. Furthermore, the multifractal character of electric field fluctuations has been shown to be different in the two investigated polar regions: the polar cap trailing edge and the auroral zone. This difference, also supported by the different values of the multifractal singularity spectrum skewness $s$, has been interpreted in terms of the different nature of plasma circulation and particle precipitation that the CSES-01 satellite experiences crossing the polar ionospheric region. This hypothesis is supported by previous studies [54] that revealed different spectral and intermittency features of plasma fluctuations in different ionospheric polar regions. The observed differences could be due to the particle precipitation in the polar cap and the auroral zone.

Last but not least, the strong roughness of the electric field fluctuations in the geomagnetic field direction has been conjectured to be due to a multifractal character of the FACs in the auroral region.

Clearly, further work is necessary to better assess the multifractal character of electric field fluctuations and their link with other physical quantities (such as FACs), so that this work has to be considered a preliminary investigation of the complex nature of the electric field fluctuations in the polar ionosphere.

Author Contributions: Conceptualization, G.C. and V.Q.; methodology, G.C., V.Q. and T.A.; formal analysis, G.C. and V.Q.; investigation, All; data curation, M.P.; writing-original draft preparation, G.C.; writing-review and editing, G.C., V.Q., G.D., T.A., M.P., M.F.M., P.D.M. All authors have read and agreed to the published version of the manuscript. 
Funding: This research is supported by the contract ASI "LIMADOU Scienza+" $\mathrm{n}^{\circ}$ 2020-31-HH.0 and Italian PNRA under contract PNRA18 00289-A "Space weather in Polar Ionosphere: the Role of Turbulence".

Institutional Review Board Statement: Not applicable.

Informed Consent Statement: Not applicable.

Data Availability Statement: This work made use of the data from the CSES mission (http:/ / www. leos.ac.cn/, acessed on 1 October 2020), a project funded by China National Space Administration and China Earthquake Administration in collaboration with Italian Space Agency and Istituto Nazionale di Fisica Nucleare. The Special Sensor Ultraviolet Spectrographic Imager (SSUSI) data are available and documented at https:/ / ssusi.jhuapl.edu/ (acessed on 1 October 2020). The SSUSI Principal Investigator is Dr. Larry J. Paxton. Magnetic data used are part of the worldwide network of observatories INTERMAGNET (http:/ / www.intermagnet.org/, acessed on 1 October 2020).

Acknowledgments: This work is in the framework of the CSES-LIMADOU Collaboration (http:/ / cses.roma2.infn.it, acessed on 1 October 2020). We acknowledge the Italian Space Agency (ASI) and the Italian National Project for Anctartic Research (PNRA) for supporting this work in the framework of contract ASI "LIMADOU Scienza+" n² 2020-31-HH.0 and PNRA18 00289-A "Space weather in Polar Ionosphere: the Role of Turbulence". V. Quattrociocchi, G. D'Angelo and M. Piersanti thank the Italian Space Agency for the financial support under the contract "LIMADOU-2 fase B2/C/D/E1". The authors acknowledge the use of SuperDARN data. SuperDARN is a collection of radars funded by national scientific funding agencies of Australia, Canada, China, France, Italy, Japan, Norway, South Africa, United Kingdom and the United States of America.

Conflicts of Interest: The authors declare no conflict of interest.

\section{Abbreviations}

The following abbreviations are used in this manuscript:

$\begin{array}{ll}\text { AACGM } & \text { Altitude Adjusted Corrected Geomagnetic coordinates } \\ \text { AE } & \text { Auroral Electrojet index } \\ \text { AL } & \text { Auroral Low electrojet index } \\ \text { CSES } & \text { Chinese Seismo-Electromagnetic Satellite } \\ \text { DMSP } & \text { Defense Metereological Satellite Project } \\ \text { EFD } & \text { Electric Field Detector } \\ \text { ELF } & \text { Extra Low Frequency } \\ \text { FAC } & \text { Field Aligned Current } \\ \text { IMF } & \text { Interplanetary Magnetic Field } \\ \text { INTERMAGNET } & \text { International Magnetic Network } \\ \text { MAW } & \text { Mawson geomagnetic observatory } \\ \text { MHD } & \text { Magneto-Hydro-Dynamics } \\ \text { MLat } & \text { Magnetic Latitude } \\ \text { MLT } & \text { Magnetic Local Time } \\ \text { MVA } & \text { Minimum Variance Analysis } \\ \text { PSD } & \text { Power Spectral Density } \\ \text { ROMA } & \text { Rank-Ordering Multifractal Analysis } \\ \text { SuperDARN } & \text { Super Dual Auroral Radar Network } \\ \text { ULF } & \text { Ultra Low Frequency } \\ \text { UT } & \text { Universal Time } \\ \text { WDC } & \text { World Data Center }\end{array}$

\section{References}

1. Kintner, P.M., Jr. Observations of velocity shear driven plasma turbulence. J. Geophys. Res. 1976, 5114-5122. [CrossRef]

2. Kintner, P.M.; Seyler, C.E. The status of observations and theory of high latitude ionospheric and magnetospheric plasma turbulence. Space Sci. Rev. 1985, 41, 1572-9672. [CrossRef]

3. Basu, S.; Basu, S.; MacKenzie, E.; Fougere, P.F.; Coley, W.R.; Maynard, N.C.; Winningham, J.D.; Sugiura, M.; Hanson, W.B.; Hoegy, W.R. Simultaneous density and electric field fluctuation spectra associated with velocity shears in the auroral oval. J. Geophys. Res. Space Phys. 1988, 93, 115-136. [CrossRef] 
4. Tam, S.W.Y.; Chang, T.; Kintner, P.M.; Klatt, E. Intermittency analyses on the SIERRA measurements of the electric field fluctuations in the auroral zone. Geophys. Res. Lett. 2005, 32, L05109. [CrossRef]

5. Golovchanskaya, I.V.; Ostapenko, A.A.; Kozelov, B.V. Relationship between the high-latitude electric and magnetic turbulence and the Birkeland field-aligned currents. J. Geophys. Res. (Space Phys.) 2006, 111, A12301. [CrossRef]

6. Golovchanskaya, I.V.; Kozelov, B.V. On the origin of electric turbulence in the polar cap ionosphere. J. Geophys. Res. (Space Phys.) 2010, 115, A09321. [CrossRef]

7. Golovchanskaya, I.V.; Kozelov, B.V. Properties of electric turbulence in the polar cap ionosphere. Geomagn. Aeron. 2010, 50, 576-587. [CrossRef]

8. Kozelov, B.V.; Golovchanskaya, I.V. Scaling of electric field fluctuations associated with the aurora during northward IMF. Geophys. Res. Lett. 2006, 33, L20109. [CrossRef]

9. Kozelov, B.V.; Golovchanskaya, I.V.; Ostapenko, A.A.; Fedorenko, Y.V. Wavelet analysis of high-latitude electric and magnetic fluctuations observed by the Dynamic Explorer 2 satellite. J. Geophys. Res. (Space Phys.) 2008, 113, A03308. [CrossRef]

10. Kintner, P.M.; Franz, J.; Schuck, P.; Klatt, E. Interferometric coherency determination of wavelength or what are broadband ELF waves? J. Geophys. Res. 2000, 105, 21237-21250. [CrossRef]

11. André, M.; Norqvist, P.; Andersson, L.; Eliasson, L.; Eriksson, A.I.; Blomberg, L.; Erlandson, R.E.; Waldemark, J. Ion energization mechanisms at $1700 \mathrm{~km}$ in the auroral region. J. Geophys. Res. 1998, 103, 4199-4222. [CrossRef]

12. Chang, T. Colloid-like Behavior and Topological Phase Transitions in Space Plasmas: Intermittent Low Frequency Turbulence in the Auroral Zone. Phys. Scr. Vol. T 2001, 89, 80-83. [CrossRef]

13. Chang, T.; Tam, S.W.Y.; Wu, C.C. Complexity induced anisotropic bimodal intermittent turbulence in space plasmas. Phys. Plasmas 2004, 11, 1287-1299. [CrossRef]

14. Tam, S.W.Y.; Chang, T.; Kintner, P.M.; Klatt, E.M. Rank-ordered multifractal analysis for intermittent fluctuations with global crossover behavior. Phys. Rev. E 2010, 81, 036414. [CrossRef]

15. Tam, S.W.Y.; Chang, T. Double rank-ordering technique of ROMA (Rank-Ordered Multifractal Analysis) for multifractal fluctuations featuring multiple regimes of scales. Nonlinear Process. Geophys. 2011, 18, 405-414. [CrossRef]

16. Abel, G.A.; Freeman, M.P.; Chisham, G. Spatial structure of ionospheric convection velocities in regions of open and closed magnetic field topology. Geophys. Res. Lett. 2006, 33, L24103. [CrossRef]

17. Abel, G.A.; Freeman, M.P.; Chisham, G.; Watkins, N.W. Investigating turbulent structure of ionospheric plasma velocity using the Halley SuperDARN radar. Nonlinear Process. Geophys. 2007, 14, 799-809. [CrossRef]

18. Kozelov, B.V.; Golovchanskaya, I.V. Derivation of aurora scaling parameters from ground-based imaging observations: Numerical tests. J. Geophys. Res. (Space Phys.) 2010, 115, A02204. [CrossRef]

19. Shen, X.; Zhang, X.; Yuan, S.; Wang, L.; Cao, J.; Huang, J.; Zhu, X.; Piergiorgio, P.; Dai, J. The state-of-the-art of the China Seismo-Electromagnetic Satellite mission. Sci. China E Technol. Sci. 2018, 61, 634-642. [CrossRef]

20. Huang, J.; Lei, J.; Li, S.; Zeren, Z.; Li, C.; Zhu, X.; Yu, W. The Electric Field Detector (EFD) onboard the ZH-1 satellite and first observational results. Earth Planet. Phys. 2018, 2, 469-478. [CrossRef]

21. Liu, C.; Guan, Y.; Zheng, X.; Zhang, A.; Piero, D.; Sun, Y. The technology of space plasma in-situ measurement on the China Seismo-Electromagnetic Satellite. Sci. China E Technol. Sci. 2019, 62, 829-838. [CrossRef]

22. Chhabra, A.; Jensen, R.V. Direct determination of the $\mathrm{f}(\alpha)$ singularity spectrum. Phys. Rev. Lett. 1989, 62, 1327-1330. [CrossRef] [PubMed]

23. Chhabra, A.B.; Meneveau, C.; Jensen, R.V.; Sreenivasan, K.R. Direct determination of the $\mathrm{f}($ alpha $)$ singularity spectrum and its application to fully developed turbulence. Phys. Rev. A 1989, 40, 5284-5294. [CrossRef] [PubMed]

24. Basu, S.; MacKenzie, E.; Basu, S.; Coley, W.R.; Sharber, J.R.; Hoegy, W.R. Plasma structuring by the gradient drift instability at high latitudes and comparison with velocity shear driven processes. J. Geophys. Res. 1990, 95, 7799-7818. [CrossRef]

25. Consolini, G.; De Michelis, P.; Alberti, T.; Giannattasio, F.; Coco, I.; Tozzi, R.; Chang, T.T.S. On the multifractal features of low-frequency magnetic field fluctuations in the field-aligned current ionospheric polar regions: Swarm observations. J. Geophys. Res. Space Phys. 2020, 125. [CrossRef]

26. Paxton, L.J.; Meng, C.I.; Fountain, G.H.; Ogorzalek, B.S.; Darlington, E.H.; Gary, S.A.; Goldsten, J.O.; Kusnierkiewicz, D.Y.; Lee, S.C.; Linstrom, L.A.; et al. SSUSI: Horizon-to-Horizon and Limb-Viewing Spectrographic Imager for Remote Sensing of Environmental Parameters; Ultraviolet Technology IV; Huffman, R.E., Ed.; Proc. SPIE: Bellingham, WA, USA, 1993; Volume 1764, pp. 161-176. [CrossRef]

27. Paxton, L.J.; Schaefer, R.K.; Zhang, Y.; Kil, H. Far ultraviolet instrument technology. J. Geophys. Res. (Space Phys.) 2017, 122, 2706-2733. [CrossRef]

28. Diego, P.; Huang, J.; Piersanti, M.; Badoni, D.; Zeren, Z.; Yan, R.; Rebustini, G.; Ammendola, R.; Candidi, M.; Guan, Y.B.; et al. The Electric Field Detector on Board the China Seismo Electromagnetic Satellite-In-Orbit Results and Validation. Instruments 2021, 5, 1. [CrossRef]

29. Greenwald, R.A.; Baker, K.B.; Dudeney, J.R.; Pinnock, M.; Jones, T.B.; Thomas, E.C.; Villain, J.P.; Cerisier, J.C.; Senior, C.; Hanuise, C.; et al. Darn/Superdarn: A Global View of the Dynamics of High-Lattitude Convection. Space Sci. Rev. 1995, 71, 761-796. [CrossRef] 
30. Chisham, G.; Lester, M.; Milan, S.E.; Freeman, M.P.; Bristow, W.A.; Grocott, A.; McWilliams, K.A.; Ruohoniemi, J.M.; Yeoman, T.K.; Dyson, P.L.; et al. A decade of the Super Dual Auroral Radar Network (SuperDARN): Scientific achievements, new techniques and future directions. Surv. Geophys. 2007, 28, 33-109. [CrossRef]

31. Nishitani, N.; Ruohoniemi, J.M.; Lester, M.; Baker, J.B.H.; Koustov, A.V.; Shepherd, S.G.; Chisham, G.; Hori, T.; Thomas, E.G.; Makarevich, R.A.; et al. Review of the accomplishments of mid-latitude Super Dual Auroral Radar Network (SuperDARN) HF radars. Prog. Earth Planet. Sci. 2019, 6, 27. [CrossRef]

32. SuperDARN Data Analysis Working Group; Thomas, E.G.; Ponomarenko, P.V.; Billett, D.D.; Bland, E.C.; Burrell, A.G.; Kotyk, K.; Reimer, A.S.; Schmidt, M.T.; Shepherd, S.G.; et al. SuperDARN Radar Software Toolkit (RST) 4.2; Zenodo: Geneva, Switzerland, 2018. [CrossRef]

33. Ruohoniemi, J.M.; Baker, K.B. Large-scale imaging of high-latitude convection with Super Dual Auroral Radar Network HF radar observations. J. Geophys. Res. 1998, 103, 20797-20811. [CrossRef]

34. Thomas, E.G.; Shepherd, S.G. Statistical Patterns of Ionospheric Convection Derived From Mid-latitude, High-Latitude, and Polar SuperDARN HF Radar Observations. J. Geophys. Res. (Space Phys.) 2018, 123, 3196-3216. [CrossRef]

35. Reiff, P.H.; Burch, J.L. IMF B ${ }_{y}$-dependent plasma flow and Birkeland currents in the dayside magnetosphere: 2 . A global model for northward and southward IMF. J. Geophys. Res. 1985, 90, 1595-1610. [CrossRef]

36. Yeoman, T.K.; Davies, J.A.; Wade, N.M.; Provan, G.; Milan, S.E. Combined CUTLASS, EISCAT and ESR observations of ionospheric plasma flows at the onset of an isolated substorm. Ann. Geophys. 2000, 18, 1073-1087. [CrossRef]

37. Mandelbrot, B.B. Multifractal measures, especially for the geophysicist. Pure Appl. Geophys. 1989, 131, 5-42. [CrossRef]

38. Sornette, D. (Ed.) Critical Phenomena in Natural Sciences; Springer: Berlin/Heidelberg, Germany, 2006.

39. Paladin, G.; Vulpiani, A. Anomalous scaling laws in multifractal objects. Phys. Rep. 1987, 156, 147-225. [CrossRef]

40. Muzy, J.F.; Bacry, E.; Arneodo, A. Wavelets and multifractal formalism for singular signals: Application to turbulence data. Phys. Rev. Lett. 1991, 67, 3515-3518. [CrossRef]

41. Frisch, U. Turbulence: The Legacy of A. N. Kolmogorov; Cambridge University Press: Cambridge, UK, 1995. [CrossRef]

42. Lagoutte, D.; Cerisier, J.C.; Plagnaud, J.L.; Villain, J.P.; Forget, B. High-latitude ionospheric electrostatic turbulence studied by means of the wavelet transform. J. Atmos. Terr. Phys. 1992, 54, 1283-1293. [CrossRef]

43. Mounir, H.; Berthelier, A.; Cerisier, J.C.; Lagoutte, D.; Beghin, C. The small-scale turbulent structure of the high latitude ionosphere-Arcad-Aureol-3 observations. Ann. Geophys. 1991, 9, 725-737.

44. Earle, G.D.; Kelley, M.C.; Ganguli, G. Large velocity shears and associated electrostatic waves and turbulence in the auroral F region. J. Geophys. Res. Space Phys. 1989, 94, 15321-15333. [CrossRef]

45. Davis, A.; Marshak, A.; Wiscombe, W.; Cahalan, R. Multifractal characterizations of nonstationarity and intermittency in geophysical fields: Observed, retrieved, or simulated. J. Geophys. Res. Atmos. 1994, 99, 8055-8072. [CrossRef]

46. Marsch, E.; Tu, C.Y.; Rosenbauer, H. Multifractal scaling of the kinetic energy flux in solar wind turbulence. Ann. Geophys. 1996, 14, 259-269. [CrossRef]

47. Boldyrev, S.; Mason, J.; Cattaneo, F. Dynamic Alignment and Exact Scaling Laws in Magnetohydrodynamic Turbulence. Asprophys. J. 2009, 699, L39-L42. [CrossRef]

48. Friedrich, J.; Homann, H.; Schäfer, T.; Grauer, R. Longitudinal and transverse structure functions in high Reynolds-number magneto-hydrodynamic turbulence. New J. Phys. 2016, 18, 125008. [CrossRef]

49. Parks, G. (Ed.) Physics of Space Plasmas; Westview Press: Boulder, CO, USA, 2004.

50. Consolini, G.; De Michelis, P.; Coco, I.; Alberti, T.; Marcucci, M.F.; Giannattasio, F.; Tozzi, R. Sign-Singularity Analysis of Ionospheric Field-Aligned Currents. Atmosphere 2021, submitted.

51. Kelley, M.C.; Kintner, P.M. Evidence for two-dimensional inertial turbulence in a cosmic-scale low-beta plasma. Astrophys. J. 1978, 220, 339-343. [CrossRef]

52. Chang, T.; Wu, C.C. Rank-ordered multifractal spectrum for intermittent fluctuations. Phys. Rev. E 2008, 77, 045401. [CrossRef] [PubMed]

53. Chang, T.; Cheng-Chin, W.; Podesta, J. Multifractal Characteristics of Dynamical Complexity in Space Plasmas. In Particle Acceleration and Transport in the Heliosphere and Beyond, Proceedings of the 7th Annual International AstroPhysics Conference, Kauai, HI, USA, 7-13 March 2008; American Institute of Physics Conference Series; Li, G., Hu, Q., Verkhoglyadova, O., Zank, G.P., Lin, R.P., Luhmann, J., Eds.; AIP Publishing: Melville, NY, USA, 2008; Volume 1039, pp. 75-80. [CrossRef]

54. Spicher, A.; Miloch, W.J.; Clausen, L.B.N.; Moen, J.I. Plasma turbulence and coherent structures in the polar cap observed by the ICI-2 sounding rocket. J. Geophys. Res. Space Phys. 2015, 120, 10959-10978. [CrossRef] 